\title{
Myanmar Continues to Curb Malaria amid Coronavirus Disease-2019 Crisis
}

\author{
Badri Thapa ( $\nabla$ badri_bishal@yahoo.com ) \\ World Health Organization https://orcid.org/0000-0003-0851-9491 \\ Aung Thi \\ National Malaria Control Programme \\ Wint Phyo Than \\ National Malaria Control Programme
}

Kyawt Mon Win

National Malaria Control Programme

San Kyaw Khine

World Health Organization Country Office for Myanmar

\section{Research}

Keywords: Malaria, COVID-19, Myanmar

Posted Date: November 9th, 2020

DOI: https://doi.org/10.21203/rs.3.rs-101547/v1

License: (c) (i) This work is licensed under a Creative Commons Attribution 4.0 International License. Read Full License 
Title page

Title

Myanmar continues to curb malaria amid Coronavirus Disease-2019 crisis

Aung Thi ${ }^{1}$, Wint Phyo Than ${ }^{1}$, Kyawt Mon Win ${ }^{1}$, San Kyawt Khine ${ }^{2}$, Badri Thapa ${ }^{2 *}$

\section{Author details}

${ }^{1}$ Naional Malaria Control Programme, Vector Borne Disease Control Programme, Myanmar;

${ }^{2}$ World Health Organization, Myanmar

*Corresponding author: Badri Thapa, World Health Organization, Myanmar, thapab@who.int 


\section{Abstract}

Background: Myanmar has targeted Plasmodium falciparum malaria elimination by 2025 and all human malaria elimination by 2030. Coronavirus Disease-2019 (COVID-19) pandemic could jeopardize the current gains. The objective of the study is to assess the impact of COVID-19 on epidemiology and malaria services in Myanmar.

Methods: This is cross sectional study using retrospective routine programme data reported by Ministry of Health and Sports (for COVID-19) and National Malaria Control Programme (NMCP) (for malaria) during January - September 2019 and 2020.

Results: Myanmar implemented whole of society COVID-19 response since its first 2 cases were detected on 23 March 2020. NMCP mobilized US\$ 7 mil to support, protect and mitigate COVID-19 impact on malaria through implementation of tailored guidelines, job aids and risk communication materials starting April 2020. Front line health workers were protected through supplies of surgical masks, hand sanitizer, aprons, gloves, non-contact digital thermometers among others. During January-September 2020, reported malaria cases, severe cases and deaths declined by $11 \%, 34 \%$ and $27 \%$, respectively in the same period in 2019 . Total $P$. falciparum cases declined by $55 \%$ with minimal increase in tests $(0.14 \%) .80 \%$ of cases and $98 \%$ of foci were investigated in 2020 in comparison to $68 \%$ and $54 \%$ in 2019, respectively $(p<0.05)$. In 2020, NMCP distributed $72 \%$ of the targeted long-lasting insecticidal nets (vs $49 \%$ in 2019) $(p<0.05)$. Integrated Community Malaria Volunteers (ICMVs) trained in 2020 was $49 \%$ in comparison to $94 \%$ in $2019(P<0.05)$, while number of functional ICMVs remained at $91 \%$ (vs $94 \%$ in 2019). Overall monthly reports from all reporting units received slightly dropped by $5 \%$. In 2020, NMCP was able to conduct $59 \%$ supervision visits at various levels, which was higher than 2019 (56\%).

Conclusions: Malaria cases, severe cases and deaths continued to decline amid COVID-19 in Myanmar with 55\% decline in P. falciparum. Programme need to continue essential malaria services during the upsurge of COVID-19 and simultaneously plan proactively to ensure uninterrupted supply of essential malaria commodities for 2021 to ensure P. falciparum elimination by 2025.

\section{Key words: Malaria, COVID-19, Myanmar}




\section{Background}

National Malaria Control Pogramme (NMCP), Myanmar has targeted Plasmodium falciparum malaria elimination by 2025 and all human malaria elimination by 2030 in line with Greater Mekong Subregion Strategy for malaria elimination 2015-2030 [1]. Over the period of 8 years, Myanmar has made an unprecedented progress in declining malaria morbidity and mortality. The cases and deaths dropped by $88 \%$ and $97 \%$, respectively in 2019 in comparison to 2012 [2]. P. falciparum cases has dropped significantly and accounted only for $41 \%$ in 2019 . Over $80 \%$ of malaria cases lies in 20 townships in the Eastern and Western border of Myanmar. Although significant progress has been made, technical and operational challenges remains, like high malaria burden in conflict affected area, difficult to reach risk population like forest goers, mobile and migrants, outdoor malaria transmission, $P$. vivax malaria relapse, and delay reporting from some areas [3]. Achievement of the $P$. falciparum malaria elimination could be hindered due to the COVID-19 crisis ongoing in the Myanmar and globally.

The first 2 cases of COVID-19 was reported on 23 March 2020 in Myanmar [4]. The cases continue to rise starting from 16 August 2020. The SARS-CoV-2 virus transmission in Myanmar is characterized by 'clusters of cases' in most areas-- experiencing cases, clustered in time, geographic location, and/or by common exposures. Recently COVID-19 incidence has sharply risen with cumulative counts almost doubling every week since 15 August 2020. Myanmar is under possibility of community transmission [5]. As of 30 September 2020, the country has conducted 267,294 tests, detected 13,373 cases and reported 309 deaths and 3,755 recoveries. Of those detected, 13,060 (98\%) were from local transmission and $313(2 \%)$ were imported cases. Clusters reported in Myanmar are related to families, markets, religious sermons and events, entertainment sites, and work sites.

The COVID-19 crisis has imposed several challenges that have compromised the country health system including the malaria health system. On 27 August 2020, WHO published a survey report assessing the impact of COVID-19 on health systems conducted in 105 countries [6]. On supply side, Ministries have repurposed malaria staff to COVID-19 tasks. Logistic procurement and supply chain disturbances have lead malaria prevention, diagnostics and drug shortages. $46 \%$ of the countries surveyed had disruptions in malaria diagnosis 
and treatment. On demand side, there is an overall decline in malaria service utilization due to fear, stigmatization and travel restrictions. In the same report, $76 \%$ of countries reported reductions in outpatient care attendance due to lower demand and other factors such as lockdowns and financial difficulties.

The Government of the Republic of the Union of Myanmar has been proactive in COVID-19 preparedness and response efforts soon after the first case of COVID-19 was reported in China in December 2019 and its first 2 cases detected on 23 March 2020. The country launched its 'Health Sector COVID-19 Contingency Plan' on 21 April 2020 with the objective to 'provide a national level contingency plan for managing comprehensive health sector response to COVID-19. It also addresses the urgent need to protect healthcare professionals and other frontline workers who are at the forefront of containing the spread of COVID-19 to protect themselves and mitigate any potential impacts. In line with the national plans and WHO recommendations, NMCP developed malaria guidelines to tailor malaria interventions amid COVID-19 [7]. NMCP and its technical and civil society partners are implementing essential malaria services amid of COVID-19 situation in the country. The current study examined an impact of COVID-19 on key malaria services and epidemiology in Myanmar.

\section{Methods}

\section{Study design}

This was a cross sectional study based on the retrospective routine programmatic data analysis on COVID-19 and Malaria in Myanmar.

\section{General setting}

The Republic of The Union of Myanmar is one of the countries in WHO South East Asia Region sharing border with India, Bangladesh, China, Laos and Thailand. It is administratively divided into 14 States and Regions and one Nay Pyi Taw Council territory with 54 million population [8]. Ministry of Health and Sports of Myanmar (MoHS) is composed of 6 departments. Disease control division and Central Epidemiology Unit (CEU) under the Department of Public Health carries out disease prevention and control activities. Integrated diseases surveillance and outbreak management are mainly carried out by CEU at central level, Special Diseases Control Units at State/Regional level and Basic Health Staffs at township and community level.

Malaria is endemic in 291 out of 330 townships in Myanmar [9]. National Malaria Control Progamme (NMCP) is under the Disease Control Division which carries out malaria control and elimination activities. Vector Borne Diseases Control teams at State/Region Health Department, Township Hospitals, Station Hospitals, Rural 
Health Care Centers and Sub-centers manage malaria programmes. Township is the operational unit for malaria control and elimination activities.

\section{Specific setting}

MoHS is actively conducting surveillance for COVID-19 throughout the country. All patient under investigation - meeting the WHO case definition for COVID-19 suspects identified in community fever clinic, health care facilities, international returnees and contact persons of positive cases are screened for COVID-19 [10]. Nasopharyngeal swabs are collected from the COVID-19 suspects and tested using RT-PCR, GeneXpert platform and by COVID-19 Antigen based tests. Laboratory confirmed cases of COVID-19 are then admitted in infectious disease hospitals identified by MoHS throughout the country. Daily COVID-19 data is reported on MoHS website as situation reports.

Malaria services are provided free of charge to all population at risk. Case detection and management services are conducted by 21,000 Integrated Community Malaria Volunteers (ICMV) at community level (under NMCP and Civil Society Organizations- CSOs), Basic Health Staffs in over 12,000 public health facilities and over 18,00 formal and informal general practitioners in private sectors. Numerous community fever clinics have been established in the Public Health Facilities (PHFs) for COVID-19 screening and treatment. Malaria preventive intervention like Long-Lasting Insecticidal Nets (LLINs) are given through Basic health staffs, Vector Borne Diseases Control programme staffs and partners. Identified malaria suspects from all sectors are then tested either by microscopy (PHFs and microscopy centers of CSOs) and malaria Rapid Diagnostic test. The treatment is given according to national treatment guideline. In 2020, 258 townships are under malaria elimination. These townships are notifying cases and conducting case investigation, foci investigation and response activities $[11,12]$. The malaria reports are submitted on monthly basis to the reporting units.

\section{Study population}

The study included all COVID-19 cases reported during March-September 2020 and malaria suspects tested and malaria cases diagnosed during January - September 2019 and 2020. Malaria case is defined as a person tested positive by RTD and/or microscopy and recorded in a patient register regardless of the presence or absence of clinical symptoms [14].

\section{Data variables and source}

Variables included in the study are aggregated numbers of monthly COVID-19 cases and malaria suspects tested, malaria cases diagnosed (disaggregated by species), serve malaria cases and death, Long-lasting 
Insecticidal nets (LLIN) distributed, physical/ virtual meeting, trainings supervision visits and reports received. Malaria variables were extracted from The Global Fund to fight AIDS, Tuberculosis and Malaria (GFATM) 6 monthly performance update reports (January-June), Malaria Web based Surveillance System, and GFATM procurement reports and COVID-19 cases were extracted from MoHS surveillance reports [15].

\section{Data analysis}

The study was a secondary data analysis of aggregate data collected and analyzed using the Microsoft Excel. Results were expressed in number and proportions. Comparison of proportion were done by Chi-square test with level of significance reported for $p<0.05$.

\section{Results}

\section{Key public health events for COVID-19 and malaria response in Myanmar}

The trends of COVID-19 and malaria cases with key public health measures mounted by Myanmar were presented in Figure 1. Myanmar started its preparedness to respond COVID-19 since it was reported on 29 December 2019 in China. Preparedness with surveillance in point of entries (airports, border crossing points and sea ports) and risk communication and community engagement started on 5 January 2020. Central level coordination committee for COVID-19 prevention, treatment and control was established on 10 February 2020. National Malaria Control Programme (NMCP) started to emphasize maintaining its interventions by developing guidelines to tailor malaria interventions amid COVID-19 and job aids starting April 2020 soon after first 2 cases of COVID-19 was reported on 23 March 2020. The funds were mobilized from existing sources and additional resource were mobilized through COVID-19 Response Mechanisms (supported by GFATM). NMCP continued its malaria essential services which are presented below.

\section{Support, protect and mitigate impact of COVID-19 on malaria}

Amid COVID-19 situation, NMCP and its partners continued essential malaria activities - test, treat, prevention and reporting. NMCP's approach to support, protect and mitigate malaria interventions amid COVID-19 is presented in Table 1 . The risk assessment informed identification of mitigation measures to deliver malaria interventions amid COVID-19. NMCP suspended most of the central and State/Regional level physical meetings - weighing the risk versus need- and replaced with virtual meetings while essential physical meetings/trainings 
and supervision visits continued with protection. Programme proactively mobilized US\$ 7 million from COVID19 Response Mechanisms from GFATM and reprogrammed savings from GFATM grant to support, protect and mitigate impact of COVID-19 in malaria. Personal protective equipment provided, and mitigation measures taken are presented in Table 1.

\section{Impact of COVID-19 on malaria during January-September 2020}

The comparison of malaria data variables between January - September 2020 and same period in 2019 is shown in Table 1 . The reported malaria cases declined by $11 \%$ in 2020 . Strikingly the maximum decline was noted in mix cases (60\%), followed by $P$. falciparum (55\%). At the same time, there was upsurge of $P$. vivax by $16 \%$. The reported severe cases declined by $34 \%$ and $27 \%$ in 2020 in comparison to 2019 , respectively. At the same time, the total malaria test in 2020 was slightly higher in 2020 (by $0.14 \%$ ) than 2019. During January June 2020, NMCP distributed 0.18 LLINs (72\% of target) through continuous channel, higher proportion than $2019(49 \%)(p<0.05)$. Case and foci investigated were $80 \%$ and $98 \%$ in 2020 , which were higher in comparison to $2019(68.4 \%$ and $54.1 \%)$, respectively ( $<<0.05)$. Amid- COVID-19 situation in 2020, programme was able to train 49\% ICMVs in comparison to $94 \%$ in $2019(p<0.05)$. In 2020, programme was able to conduct 59\% supervision visits at various levels, which was higher than 2019 (56\%) but not significantly different ( $p>0.05)$. Overall monthly reports received was $81 \%$ in 2020 in comparison to $86 \%$ in 2019 , with slight drop of 5\% $(p<0.05)$. While number of functional ICMVs (reporting quarterly) remained at the same level during the two periods $(2019,94 \%$ and $2020,91 \%)(p<0.05)$.

\section{Overlap of malaria and COVID-19}

Figure 2 shows geographical overlap between Annual Parasite Incidence (API) per 1,000 population at risk and COVID-19 cases by States/Regions. Malaria transmission is heterogenous in States/Region in Myanmar as shown by the intensity of transmission. Of 15 States/Regions, 14 (except Kayah) have no reported COVID-19 case up to 30 September 2020 . Over $75 \%(10,085)$ of reported cases have been reported from Yangon Region, followed by Rakhine $(12 \%, 1,568)$, Bago $(3 \%, 386)$ and Ayeyarwaddy $(2 \%, 310)$. In 2019, Chin State had the highest Annual Parasite Incidence per 1,000 population at risk (34.76) followed by Kayin (8.67), Tanintharyi (4.91), Kachin (3.08) and Rakhine (2.44). COVID-19 cases overlapped with malaria in Rakhine. In contrary, highest number of COVID-19 cases were seen in Yangon with lowest malaria transmission (API, 0.01). 


\section{Discussion}

The current study assessed the impact of COVID-19 on malaria in Myanmar at a national scale during JanuarySeptember 2020. Myanmar reported 13, 373 cases and 309 deaths of COVID-19 at the end of September 2020. During January - September 2020, malaria cases declined by $11 \%$ in comparison to 2019 . The severe cases and deaths also declined by $34 \%$ and $27 \%$ respectively. NMCP mobilized US\$ 7 million support, protect and mitigate the impact of COVID-19 on malaria services. Essential malaria services like, test- treat-track, prevention (LLINs distribution) and reporting continued with mitigation measures- promotion of COVID-19 health literacy, personal protective equipment for frontline malaria workers- as per the COVID-19 epidemiology and Government's COVID-19 response guidelines. It was evident that despite of COVID-19, malaria cases and deaths declined during January-September 2020.

This is the first study conducted to assess the impact of COVID-19 on malaria under NMCP in Myanmar. This has presented good lessons for NMCP, Myanmar and NMCPs of other countries that comprehensive and tailored programme service delivery could still yield good results amid pandemic. The study included all COVID19 cases reported (March - September 2020) from the routine reports from Ministry of Health and Sports and malaria cases (January - September 2020 and 2019) reported from NMCP. Use of routine data is the strength to this study.

NMCP developed its guideline to tailor malaria intervention amid COVID-19 and guided sub-national programme to continue maintaining essential services. This was in line with the essential services identified by WHO [15]. Malaria like other diseases faced numerous challenges amid COVID-19. For test-treat of malaria, health care workers require additional resources to protect themselves from COVID-19 [16]. These fears are compounded by contributing factors, including overwhelmed health facilities, fear of contracting disease at health services and deaths of health care workers. These were evidenced in Ebola crisis $[17,18]$ and is also relevant to COVID-19. During the Ebola fever crisis in West Africa, malaria deaths outnumbered total deaths 
from Ebola itself [19]. Disruptions to malaria services have been linked to over 75 major resurgences in the past [20].

Protection of health care workers from all sectors was important intervention taken by NMCP and its implementing partners. NMCP's 70\% of the malaria cases are contributed by network of ICMVs- and continued engagement of this network was crucial. NMCP timely mitigated COVID-19 impacts through development of the timely guidelines to 'tailor malaria interventions amid COVID-19' and job aids to the malaria front line workers including ICMVs. The findings have showed that there are minimal overlaps between COVID-19 and malaria. Over $90 \%$ of the COVID-19 cases are present in commercial city of Yangon Region (75\%) and urban and peri-urban areas of other States/Regions (Rakhine, Mandalay, Ayeyarwaddy). As mentioned, fear of COVID-19 could impact the access and service delivery irrespective of the locations. NMCP's timely decision to suspend physical gathering and supervision and monitoring visits and replacement with virtual meetings and follow ups in areas impacted by COVID-19 guided sub-national programmes. In contrary, continuation of the physical meetings and supervision and monitoring visits in areas less impacted by COVID-19 supported the continuation of essential malaria services. During January-June 2020, significant batches of capacity building initiatives were conducted to train numbers of VBDC staff, BHS, and ICMVs. NMCP was able to conduct 59\% supervision visits at various levels, which was higher than 2019 (56\%). This is also a clear indication that essential services continued.

WHO's Malaria Atlas Project modelled impacts of cessation of Insecticide Treated bed Nets (ITNs) distribution campaigns planned for 2020, reductions of routine ITN distribution and reduced access to effective antimalarial drugs in Sub Saharan African countries [21]. The model predicted a result of $22 \%$ increase in malaria cases and doubling of malaria deaths within a year, under a worst-case scenario-- 75\% decrease in ITN distribution coupled with $75 \%$ decrease in access to artemisinin combination therapies (Artemisinin Combination Therapies). Myanmar remained immune to this prediction and continued to decline cases and deaths. It delivered 4.5 LLINs through mass distribution in 2019- not requiring mass distribution in 2020. NMCP has already achieved $72 \%$ ( 0.18 million LLINs) of its target for continuous LLIN distribution during January-June 2020. Similarly, diagnostics (malaria rapid diagnostic tests, reagents for malaria microscopy) and antimalarials are already procured for 2020. NMCP and its partners also smartly identified savings from the ongoing grants and mobilized additional funds to support the procurement of personnel protective equipment. The funds and commodities supported NMCP to continue its core interventions amid COVID-19. 
The country target for malaria elimination is by 2030 while $P$. falciparum malaria elimination is 2025 . Malaria cases, severity and deaths are declining amid COVID-19. It is crucial for Myanmar to remain engaged for reducing the burden of malaria cases and most importantly eliminate $P$. falciparum malaria in the country to achieve its 2025 target. Amid COVID-19, malaria intensification plans are vivid in 6 high burden townships. NMCP has hired additional township epidemiologists to accelerate burden reduction in these townships. As evidenced, in the study, malaria cases continue to decline by $11 \%$. P. falciparum has declined by $55 \%$ with upsurge of $P$. vivax cases. Myanmar enforced temporary closures of the factories and business that impacted closures of banana plantation sites in high transmission sites of Kachin state (northern states) which resulted in population movements. This gave rise to unusual seasonal upsurge of $P$. vivax outbreak in few areas Kachin (data not reported). Outbreaks were due to such population movements due to natural disasters, and disease outbreaks [22].

NMCP is also proactively, continuing its elimination activities amid COVID-19. Case investigation was $80 \%$ and foci investigation was $98 \%$ in 2020 , which were higher in comparison to 2019 . At the same time, NMCP received $81 \%$ of monthly reports from all service points in 2020 in comparison to $86 \%$ in 2019 . Pending reports for 2020 are for September - which marks low transmission season in Myanmar-- which is expected not to add significant cases in September 2020.

Finding of the study should be interpreted with caution with following facts in mind: i) the data on the programmatic activities are reported based on the GFATM 6 monthly reports (January-June 2020). The interventions for July-September are not included as these will be reported by January 2021, and ii) malaria epidemiological figures are reported for January - September 2020 with reporting rate of $81 \%$-- some malaria case figures are pending. The investigators have made their efforts to include all epidemiological figures in the study.

COVID-19 cases in Myanmar are showing increasing trend and reaching to other rural locations [14]. This could potentially impact malaria activities in the remote communities. NMCP needs to remain vigilant and continue to carry out its core interventions while protecting its frontline health workers. Since COVID-19 is increasing in Myanmar, States/Regions need to prepare to tackle malaria outbreak. Bolder steps are needed to prevent upsurge of malaria in areas of low transmission in upcoming waves of COVID-19. NMCP programme officials need to remain engaged as a member of the COVID-19 response team at Central and States/Regions. Political 
commitment to maintain essential malaria services need to remain high in the agenda parallel to COVID-19.

This commitment should commensurate with mobilization of domestic funds if needed and balanced repurposing of malaria workers for COVID-19 response. In a situation where funding is secured from GFATM for 2021-2023 a proactive procurement of diagnostics, antimalarials, and LLINs is advocated for 2021. Procurement and supply chain system should be responsive to prevent stock outs amid COVID-19 global supply chain disturbances. NMCP and partners need to continue to promote health literacy on COVID-19, promotion of use protective gears and motivation to front line health care workers to main essential malaria service delivery.

\section{Conclusions}

Malaria cases and deaths continued to decline amid COVID-19 in Myanmar. This is a lesson learnt for other countries and global malaria communities. Programme need to proactively continue essential malaria services during the upsurge of COVID-19 and simultaneously plan proactively to ensure uninterrupted supply of essential malaria commodities and services in 2021.

\section{List of abbreviation}

API: Annual Parasite Incidence; BHS: Basic Health Staff; C19RM: Covid-19 Response Mechanism; CEU: Central Epidemiological Unit; COVID-19: Corona Virus Disease 2019; CSO: Civil Society Organization; GFATM: Global Fund for AIDS, Tuberculosis and Malaria; HCF: Health Care Facilities; ICMV: Integrated Community Malaria Volunteer; LLIN: Long-Lasting Insecticidal Net; M \& E: Monitoring and Evaluation; MOHS: Ministry of Health and Sports; NMCP: National Malaria Control Programme; PHF: Public Health Facilities; PUDR: Progress Update

Donor Report; RDT: Rapid Diagnostic Test; RT-PCR: Real Time- Polymerase Chain Reaction; SDCU: Special Disease Control Unit; SEARO: South East Asia Regional Office; VBDC: Vector Borne Disease Control; WHO: World Health Organization.

\section{Acknowledgements}

Authors would like to acknowledge national programme and partners for implementing malaria services amid COVID-19.

\section{Authors' contributions}


AT, WPT, KMW, SKK and BT conceived and designed the study. BT and SKK collected and preformed the data analysis. AT, WPT, KMW, SKK and BT wrote the paper. All authors read and approved the final manuscript.

\section{Funding}

None.

\section{Availability of data and materials}

The datasets generated and/or analysed during the current study are available in WHO Web site (www.who.int), Ministry of Health and Sports, Myanmar website ( mohs.gov.mm), Malaria Web-based surveillance System (data on request), and Global Fund Performance Update Reports (available on request).

\section{Ethics approval and consent to participate}

Not applicable.

\section{Consent for Publication}

Not applicable.

\section{Competing interests}

The authors declare that they have no competing interests.

\section{References}

1. WHO. Strategy for malaria elimination in Greater Mekong Sub-region (2016-2030). Geneva: World Health Organization; 2015.

2. VBDC: Vector Borne Disease Control Programme Annual Report 2019. Nay Pyi Taw: Department of Public Health, Ministry of Health and Sports, Myanmar; 2020.

3. WHO MMEP: Bulletin 8, Countries of the Greater Mekong in on falciparum malaria: Geneva: World Health Organization, Mekong Malaria Elimination Programme; 2019.

4. WHO: Corona virus disease 2019 (COVID-19) Situation report-64. https://www.who.int/docs/defaultsource/coronaviruse/situation-reports/20200324-sitrep-64-covid-19.pdf?sfvrsn=723b221e 2 Accessed 10 October 2020.

5. WHO: Coronavirus disease (COVID-19), Global Epidemiological Situation. https://www.who.int/docs/default-source/coronaviruse/situation-reports/20201005-weekly-epiupdate-8.pdf . Accessed 10 October 2020. 
6. WHO: Pulse survey on continuity of essential services during COVID-19 pandemic. Geneva: World Health Organization. 2020. https://www.who.int/publications/i/item/WHO-2019-nCoV-EHS_continuity-survey2020.1. Accessed 6 Oct 2020.

7. NMCP: Tailoring Malaria interventions in the context of COVID-19 in Myanmar: Nay Pyi Taw: Department of Public Health, Ministry of Health and Sports; 2020.

8. Myanmar Population (2020). https://www.worldometers.info/world-population/myanmar-population/ . Accessed 10 October 2020.

9. NMCP. National Strategic Plan for intensifing Malaria control and Accelerating Progress towards Malair Elimination (2016-2020). Nay Pyi Taw: Department of Public Health, Ministry of Health and Sports; 2016.

10. MOHS. Revised guideline and Standard Operating Procedure for Rapid diagnostic Test for COVID-19. Nay Pyi Taw: Ministry of Health and Sports, Myanmar; October 2020.

11. Kyaw AMM, Kathirvel S, Das M, Thapa B, Linn NYY, Maung TM, et al. "Alert-Audit-Act": Assessment of surveillance and response strategy for malaria elimination in three low-endemic settings of Myanmar in 2016. Trop Med Health. 2018;46:1-9.

12. NMCP. Malaria surveillance in elimination setting, Operational Manual. Nay Pyi Taw: Department of Public Health, Ministry of Health and Sports; 2018.

13. WHO. WHO malaira terminology. Geneva: World Health Organization; 2016.

14. MOHS. Coronavirus diesase 2019 (COVID-19) Situation reprots (Myanmar).https://mohs.gov.mm/page/9575 Accessed 10 Oct 2020.

15. WHO. Maintaining essential health services: operational guidance for the COVID-19 context. Geneva; World Health Organization; 2020.

16. Rogerson JS, Beeson JG, Laman M, Poespoprodjo JR, William T, Simpson JA, et al. Identifying and combating the impacts of COVID-19 on malaria. BMC Medicine.2020; doi: https: 10.1186/s12916-020-01710-x

17. Parpia AS, Ndeffo-Mbah ML, Wenzel NS, Galvani AP. Effects of response to 2014-2015 Ebola outbreak on deaths from malaria, HIV/AIDS, and tuberculosis, West Africa. Emerg Infect Dis. 2016;22:433-41.

18. Hamel MJ, Slutsker L. Ebola: the hidden toll. Lancet Infect Dis. 2015;15:756-7.

19. Walker PG, White MT, Griffin JT, Reynolds A, Ferguson NM, Ghani AC. Malaria morbidity and mortality in Ebola-affected countries caused by decreased health-care capacity, and the potential effect of mitigation strategies: a modelling analysis. Lancet Infect Dis. 2015;15:825-32. 
20. Cohen JM, Smith DL, Cotter C, Ward A, Yamey G, Sabot OJ, et al. Malaria resurgence: a systematic review and assessment of its causes. Malaria Journal. 2012; doi: 10.1186/1475-2875-11-122

21. World Health Organization. The potential impact of health service disruptions on the burden of malaria: a modelling analysis for countries in sub-Saharan Africa. Geneva: World Health Organization; 2020.

22. P. Martens and L. Hall. Malaria on the move: human population movement and malaria transmission. Emerg Infect Dis. 2000;6:103-09.

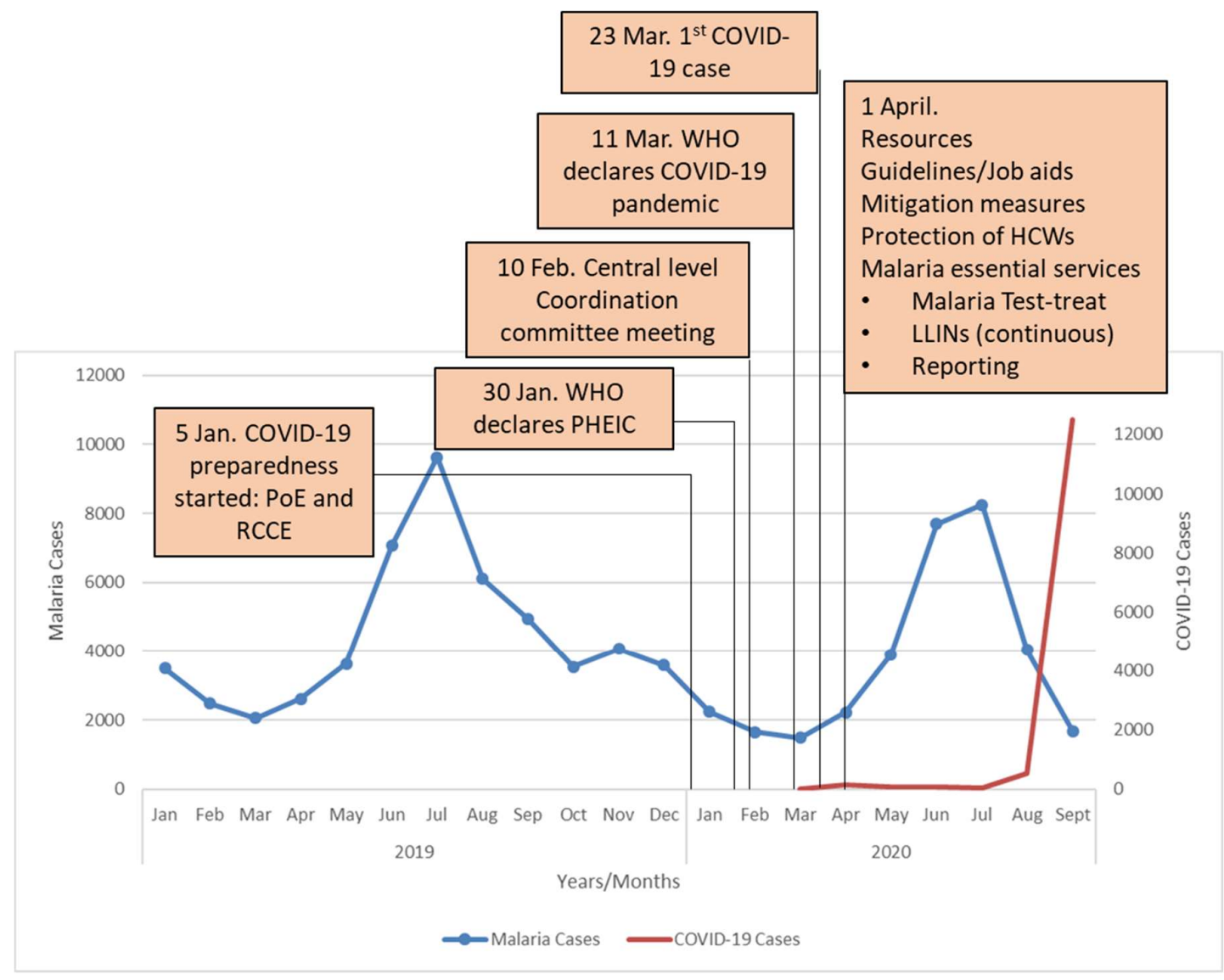

Figure 1 Monthly trend of malaria (January 2019 - September 2020) and COVID-19 (March-September 2020) confirmed cases in Myanmar with key public health events. 


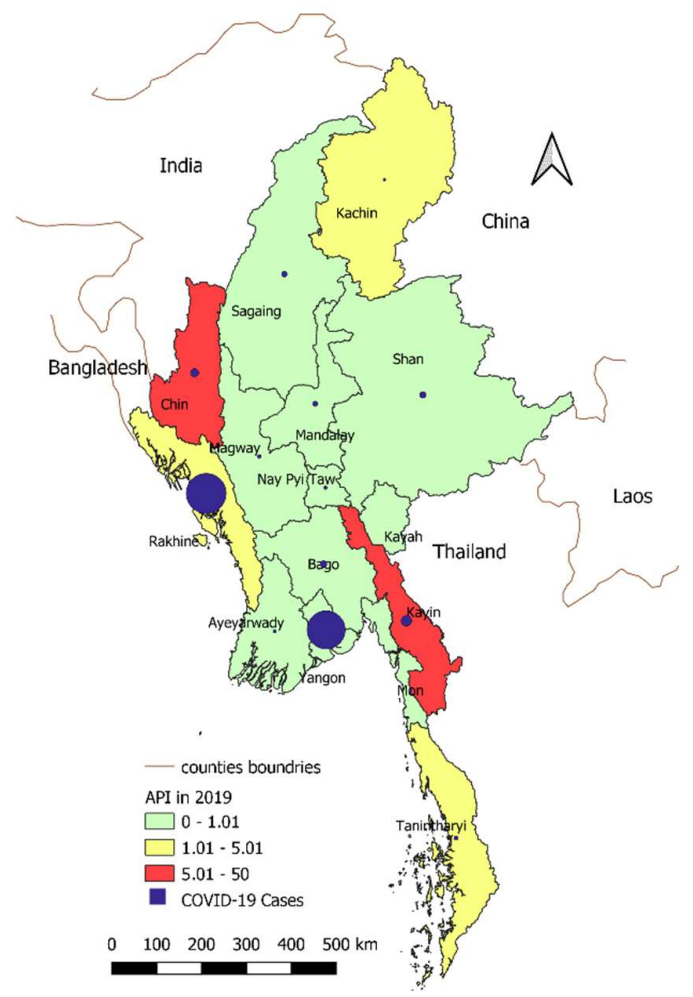

Figure 2 Overlaps of COVID-19 case load (March-September 2020) and malaria Annual Parasite Incidence (2019) in Myanmar 
Table 1 National Malaria Control Programme's approach to support, protect and mitigate malaria interventions amid COVID-19, January - September 2020, Myanmar

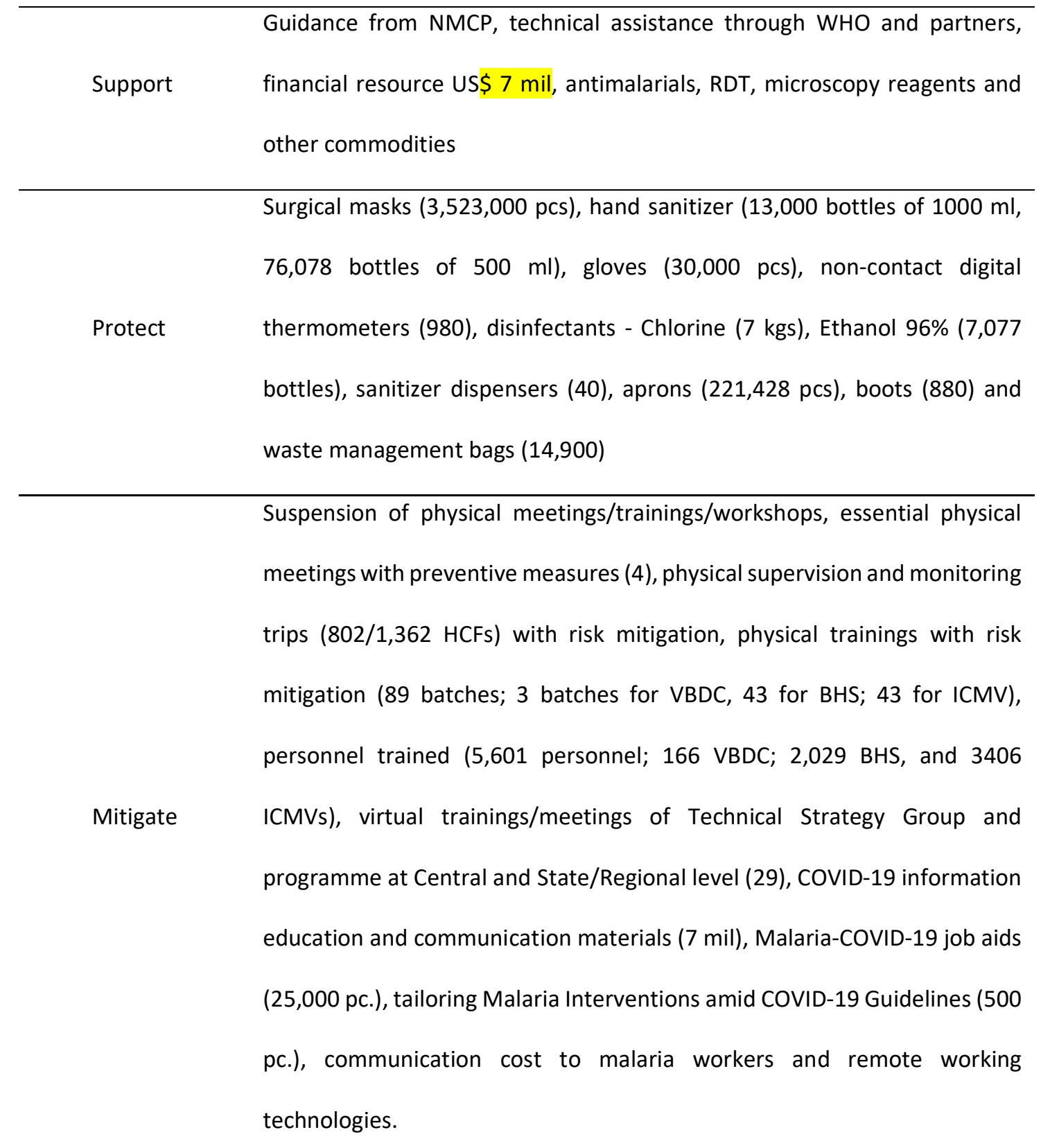

* RDT, Rapid Diagnostic test for malaria; VBDC, Vector Borne Disease Control Staff; BHS, Basic Health Staff; ICMV, Integrated Community Malaria Volunteer; mil, million; HCF, Health Care facility. Antimalarials, RDT, microscopy reagents and other commodities were procured enough for 2020. Gloves presented are in addition to supplied for testing. Mitigation activities are presented here for NMCP only. 
Table 2 Comparison of malaria cases and key interventions during January- September 2019 versus 2020

\begin{tabular}{|c|c|c|c|c|c|}
\hline \multirow{2}{*}{ Variables } & \multicolumn{2}{|c|}{2019} & \multicolumn{2}{|c|}{2020} & \multirow{2}{*}{$\%$ Reductior } \\
\hline & Target, $\mathrm{n}$ & Result, n(\%) & Target, $\mathrm{n}$ & Result, $\mathrm{n}(\%)$ & \\
\hline Total tests & - & $2,326,652$ & - & 2329877 & 0.1 \\
\hline Total malaria Cases & - & 37,044 & - & 33,121 & 11 \\
\hline P. falciparum & - & 13,494 & - & 6,026 & 55 \\
\hline P. vivax & - & 23,119 & - & 26,922 & -16 \\
\hline Mix & - & 431 & - & 173 & 60 \\
\hline Severe malaria cases & - & 90 & - & 59 & 34 \\
\hline Malaria Deaths & - & 11 & - & 8 & 27 \\
\hline LLIN distributed (continuous) & 529,391 & $258662(49)$ & 249,002 & $178755(72)$ & - \\
\hline $\begin{array}{l}\text { Confirmed cases fully } \\
\text { investigated and classified }\end{array}$ & 1,036 & 709 (68) & 1,241 & $994(80)$ & - \\
\hline $\begin{array}{l}\text { Malaria foci fully investigated } \\
\text { and classified }\end{array}$ & 303 & $164(54)$ & 223 & $218(98)$ & - \\
\hline $\begin{array}{l}\text { Reporting units submitting } \\
\text { timely monthly reports }\end{array}$ & 121,157 & $103918(86)$ & 125,203 & $101162(81)$ & - \\
\hline ICMVs trained & 2500 & $2350(94)$ & 2500 & $1220(49)$ & - \\
\hline Functioning ICMVs & 8550 & $8033(94)$ & 8550 & 7777 (91) & - \\
\hline $\begin{array}{l}\text { Supportive supervision and } \\
\text { monitoring trips }\end{array}$ & 1362 & $768(56)$ & 1362 & 802 (59) & - \\
\hline
\end{tabular}

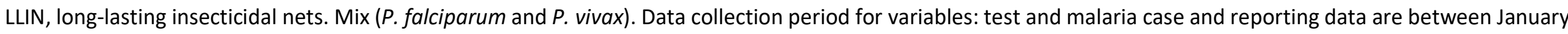

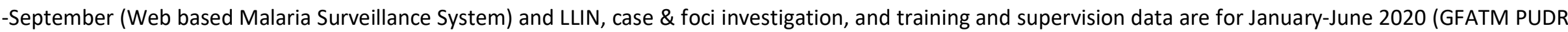
Reports). Chi-square test for difference in proportion are $<0.05$ for all comparisons except 'supportive supervision and monitoring trips'. 


\section{Figures}

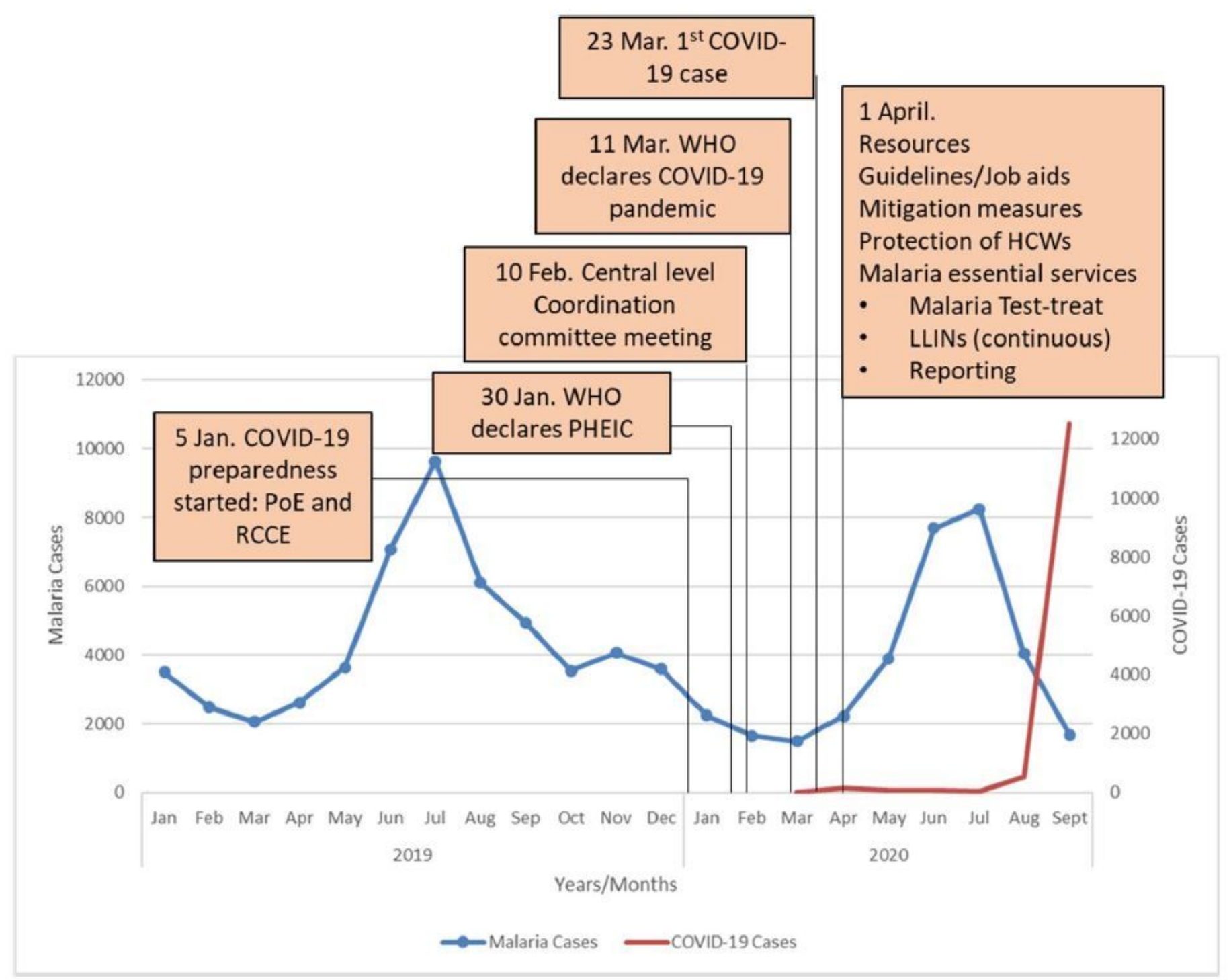

\section{Figure 1}

Monthly trend of malaria (January 2019 - September 2020) and COVID-19 (March-September 2020) confirmed cases in Myanmar with key public health events. 


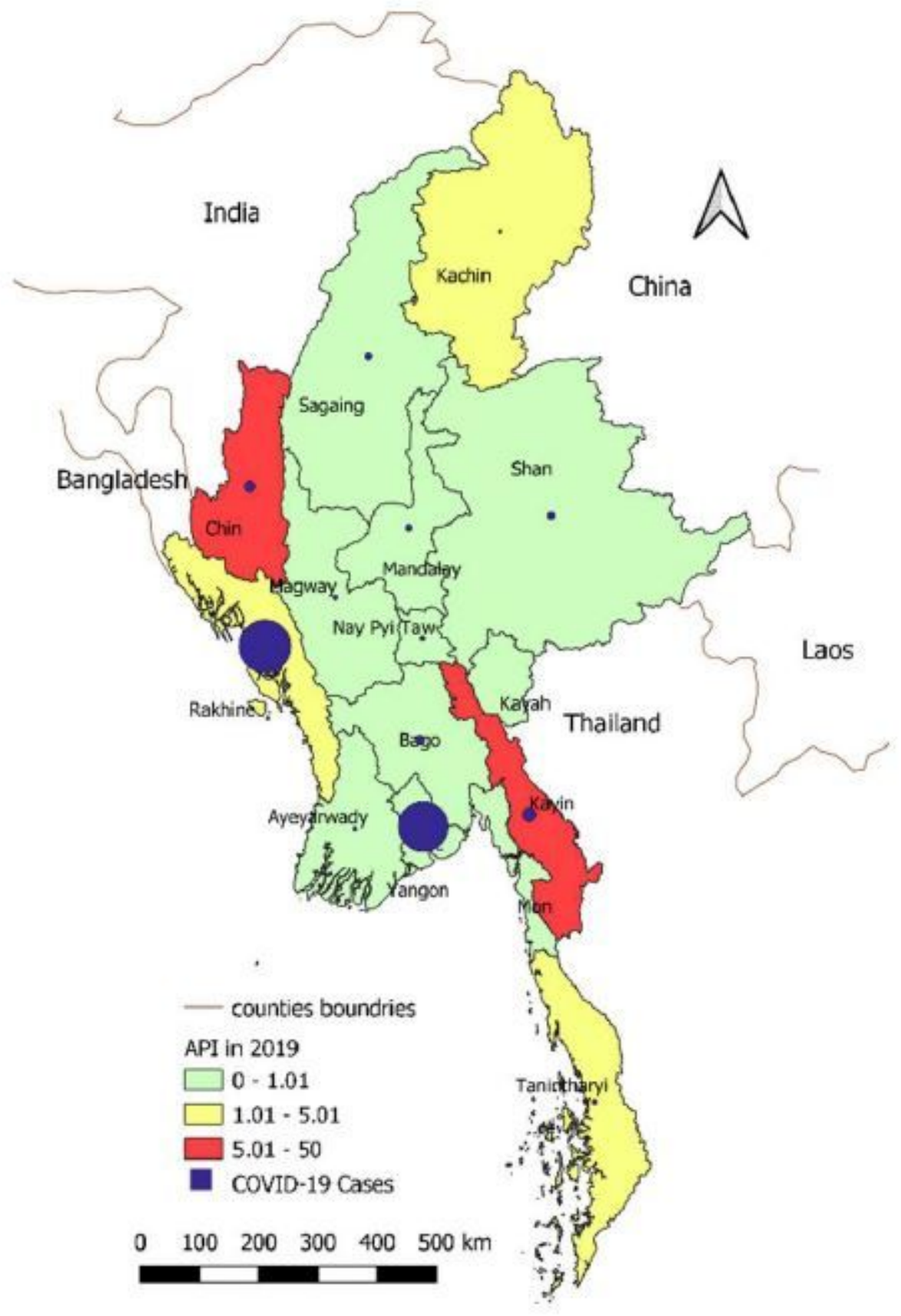

Figure 2

Overlaps of COVID-19 case load (March-September 2020) and malaria Annual Parasite Incidence (2019) in Myanmar Note: The designations employed and the presentation of the material on this map do not imply the expression of any opinion whatsoever on the part of Research Square concerning the legal status of any country, territory, city or area or of its authorities, or concerning the delimitation of its frontiers or boundaries. This map has been provided by the authors. 